\title{
Correlação espacial do índice de vegetação (NDVI) de imagem Landsat/ETM + com atributos do solo
}

\author{
Fabricio V. Zanzarini ${ }^{1}$, Teresa C. T. Pissarra ${ }^{1}$, Flavia J. C. Brandão' ${ }^{1}$ \& Daniel D. B. Teixeira ${ }^{1}$
}

\section{RESUMO}

As tecnologias de agricultura de precisão, como o uso da variabilidade espacial dos atributos do solo, vêm sendo muito estudadas para a cultura de cana-de-açúcar. Dentre essas tecnologias se destaca o uso de índices de vegetação derivados de produtos de sensoriamento remoto, como poderosas ferramentas indicadoras do desenvolvimento da vegetação. Objetivou-se, neste estudo, analisar a variabilidade espacial dos teores de argila, fósforo e o pH de um Latossolo Vermelho-amarelo em área com produção de cana-de-açúcar e correlacioná-los com o índice espectral de vegetação (NDVI). Foram estudados, a partir de uma malha georreferenciada, os atributos do solo (argila, fósforo e $\mathrm{pH}$ ) e gerados mapas de variabilidade espacial. Para esses mesmos locais calculou-se o NDVI possibilitando, além do mapeamento deste índice, a avaliação da correlação espacial entre este e as demais propriedades em estudo. A argila e o teor de fósforo apresentaram correlação espacial positiva com o NDVI enquanto nenhuma correlação espacial foi observada com o pH. A imagem do sensor ETM + do satélite Landsat 5 utilizada neste estudo em relação ao NDVI, apresentou boa aplicação para observar a variabilidade espacial dos atributos estudados.

Palavras-chave: sensoriamento remoto, variabilidade espacial, argila, $\mathrm{pH}$, fósforo

\section{Spatial correlation of the vegetation index (NDVI) of a Landsat/ETM + images with soil attributes}

\begin{abstract}
The precision agriculture technologies such as the spatial variability of soil attributes have been widely studied mostly with sugarcane. Among these technologies have been recently highlighted the use of the vegetation index derived from remote sensing products, such as powerful tools indicating the development of vegetation. This study aimed to analyze the spatial variability of clay content, $\mathrm{pH}$ and phosphorus in an Oxisol in an area with sugarcane production, and correlate with the Normalized Difference Vegetation Index (NDVI). The georeferenced grid was created for the soil properties (clay, phosphorus and $\mathrm{pH}$ ) and generated the maps of spatial variability. For these same sites were calculated the NDVI, in addition to mapping of this ratio, the evaluation of the spatial correlation between this and other studied properties. The clay and phosphorus content showed positive spatial correlation with the NDVI, while no spatial correlation was observed between NDVI and $\mathrm{pH}$. The satellite images from the sensor ETM + Landsat were used to correlate to NDVI to observe the spatial variability of the studied attributes.
\end{abstract}

Key words: remote sensing, spatial variability, clay, $\mathrm{pH}$, phosphorus 


\section{INTRODUÇÃO}

No Brasil a crescente expansão da cultura da cana-de-açúcar destaca sua importância e relevância no cenário nacional. A área colhida em 2011 ultrapassou os 9 milhões de hectares e a estimativa para 2012 é de 11 milhões de hectares. A produção brasileira subiu de 88,92 milhões para 686,29 milhões de toneladas no período de 1975 a 2009 e a produção estimada para 2012 é de 800,12 milhões de toneladas, com produtividade média passando de 46,82 para 79,76 toneladas por hectare no período de 1975 a 2009 (IBGE, 2012).

A cana-de-açúcar se desenvolve melhor em solos de boa fertilidade, profundos, argilosos, com boa capacidade de retenção de água mas sem encharcamento e com pH de 6,0 a 6,5. Apesar de ser uma planta rústica, a viabilidade econômica da cultura da cana-de-açúcar diminui à medida em que as características do solo se tornam menos favoráveis, como ambientes sujeitos a inundações ou altamente erodíveis ou declivosos ou, ainda, pedregosos e os solos de fertilidade muito baixa.

Entender como a distribuição espacial dos atributos físicos e químicos dos solos funciona, é importante para o estabelecimento de práticas de manejo adequadas na cultura de cana-de-açúcar visando não somente à otimização da produtividade agrícola mas também a minimização de possíveis danos ambientais (McBratney \& Pringle, 1999).

A análise da variabilidade do solo pode indicar, por meio da geoestatística, alternativas de manejo não só para reduzir os efeitos da variabilidade do solo sobre a produção das culturas (Trangmar et al., 1985), mas também para aumentar a possibilidade de estimar respostas das culturas sob determinadas práticas de manejo. As análises geoestatísticas se baseiam na teoria das variáveis regionalizadas em que medidas mais próximas tendem a ser mais parecidas do que valores observados em locais mais distantes (Vieira, 2000). Tais técnicas fornecem métodos para quantificar esta autocorrelação espacial e incorporá-la na estimação de valores em locais não observados (Johnsen et al., 1996). Este conjunto de ferramentas, denominado geoestatística, conduz uma das principais vertentes da chamada Agricultura de Precisão.

Outra tecnologia muito utilizada na agricultura de precisão se baseia no conhecimento de como a vegetação processa a radiação eletromagnética, técnica esta realizada por meio do sensoriamento remoto (Ponzoni, 2001). A análise da vegetação e a detecção de mudanças são realizadas com o intuito de avaliar os recursos naturais e monitorar a cobertura vegetal. Assim, o aprimoramento do conhecimento da interação da resposta eletromagnética e a vegetação, têm ocorrido pela realização de estudos no tema referido por Comportamento Espectral da Vegetação. Este fato representa as características de reflectância da resposta eletromagnética pelas folhas, plantas individuais e conjunto de plantas (dossel) (Verhulst et al., 2009; Weir \& Hering, 2012; Johnson \& Trout, 2012).

Índices espectrais de vegetação ou simplesmente índices de vegetação, têm sido largamente utilizados para monitorar a cobertura vegetal da Terra em escalas global e/ou local (Miura et al., 2001). Tais índices são combinações de dados espectrais de duas ou mais bandas, selecionadas com o objetivo de sintetizar e melhorar a relação desses dados com os parâmetros biofísicos da vegetação. Para minimizar a variabilidade causada por fatores externos a reflectância espectral tem sido transformada e combinada em vários índices de vegetação (Ponzoni, 2001).

$O$ índice de vegetação mais difundido é o Normalized Difference Vegetation Index (NDVI) (Cohen et al., 2003; Dorigo et al., 2007). Este índice pode ser analisado por meio da interpretação de imagens de sensores remotos e, em particular, por valores obtidos em diferentes datas, que permitem avaliar a variação da área verde em certo período de tempo.

Neste contexto, o principal objetivo do trabalho foi analisar o uso de uma imagem do sensor ETM+ do satélite Landsat 5 para observar a variabilidade espacial do índice de vegetação da diferença normalizada (NDVI) e avaliar se existe correlação espacial com os índices aos teores de argila, fósforo e pH do solo.

\section{Material e Métodos}

A área de estudo se localiza no município de Itajobi, SP, cujas coordenadas centrais são $49^{\circ} 05^{\prime} 29^{\prime \prime}$ W e $21^{\circ} 21^{\prime} 26^{\prime \prime}$ $\mathrm{S}$ (Figura 1A) com aproximadamente 32 ha cultivados com cana-de-açúcar. O clima da região é o tropical chuvoso, tipo Aw, segundo a classificação climática de Köeppen, caracterizado por inverno seco e verão chuvoso.

A.

B.

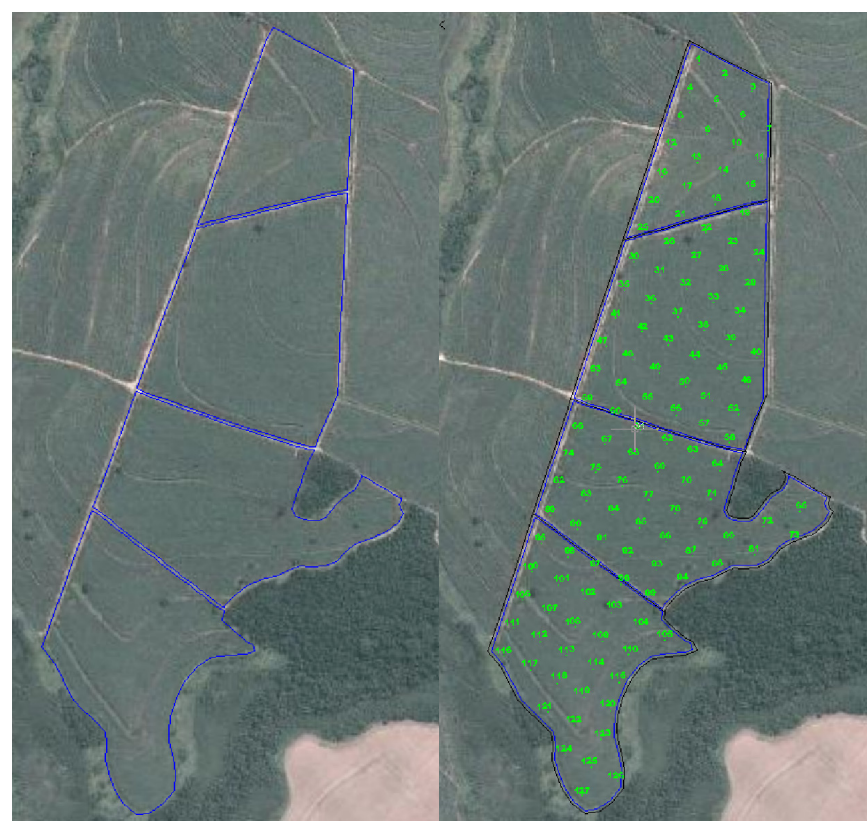

Figura 1. Imagem da área de estudo: Delimitação dos talhões estudados (A), Grid com indicação dos pontos amostrais (B)

De acordo com a caracterização pedológica pré-existente o solo é de textura predominantemente arenosa ocorrendo, porém, variações nos teores de argila, ao longo da topossequência. O solo pertence ao grupo dos Latossolos Vermelho-Amarelos textura média, distrófico. A topografia do local é plana a suavemente ondulada na maior parte da área e a declividade média é de aproximadamente 5\%.

A área de estudo é composta por 4 talhões com cultivo de cana no $5^{\circ}$ corte, totalizando 31,76 ha (Figura 1A). A variedade 
de cana-de-açúcar cultivada na área é a SP83-2847 de colheita tardia (colheita de julho a outubro). A cultura foi implantada após preparo convencional do solo no qual foram feitas todas as correções e adubações rotineiras. O plantio ocorreu no dia 13 de março de 2006; por se tratar de uma variedade de 18 meses, a primeira colheita foi no dia 20 de outubro de 2007 , sendo o segundo corte em 20/11/2008, o terceiro em 31/10/2009 e o quarto em 10/10/2010; o último corte ocorreu entre 19 e $24 / 08 / 2011$. O processo de colheita da cana foi manual, com queima da palhada.

Nos meses de novembro e dezembro de 2010 a área foi demarcada e estaqueada com auxílio de um teodolito eletrônico, em forma de grade regular de $50 \times 50 \mathrm{~m}$ gerando 124 pontos que foram georreferenciados com GPS de navegação (Figura 1B).

Ao redor de cada estaca foram coletadas, com trado holandês na profundidade de 0 a $0,10 \mathrm{~m}, 5$ amostras cada uma distanciada cerca de $2 \mathrm{~m}$; todas elas foram colocadas em um balde e homogeneizadas para formar uma amostra representativa do ponto; em seguida, foram levadas ao laboratório e aí secadas ao ar e peneiradas $(2 \mathrm{~mm})$.

Foram feitas as análises químicas para determinação de fósforo (P) e $\mathrm{pH}$ em $\mathrm{CaCl}_{2}$, no Laboratório de Análise de Solo do Departamento de Solos e Adubos da FCAV/UNESP, Jaboticabal. A composição granulométrica foi determinada para as mesmas amostras, por meio da dispersão com $\mathrm{NaOH}(0,1$ mol L-1) e agitação lenta por $16 \mathrm{~h}$; enfim o conteúdo de argila foi obtido pelo método da pipeta.

Para se calcular o índice de vegetação (NDVI) a imagem utilizada foi a do satélite Landsat 5 equipado com sensor ETM+ (Enhanced Thematic Mapper), por apresentar bandas que cobrem as principais feições da curva espectral dos vegetais, com cenas suficientemente abrangentes $\left(32.400 \mathrm{~km}^{2}-180\right.$ por $180 \mathrm{~km}$ ), por serem viáveis do ponto de vista econômico para utilização em larga escala e devido à sua qualidade radiométrica reconhecida. Neste estudo foi utilizada uma imagem da órbita/ ponto $221 / 75$, referente ao dia 24/07/2011 durante o período de crescimento vegetativo da cana nos talhões avaliados no trabalho. Todo o processamento da imagem e o cálculo do índice NDVI foram feitos utilizando-se o aplicativo ESRI ArcGIS Desktop versão 9.3. A análise foi realizada em apenas um ciclo da cultura no intuito de se avaliar a correlação com atributos do solo no estágio correspondente ao desenvolvimento da variedade estudada.

Inicialmente, o índice de vegetação da diferença normalizada referido por band ratio parameter (BRP) e hoje denominado NDVI, é calculado pela diferença entre as bandas do Infra Vermelho Próximo e do Vermelho, normalizada pela soma das mesmas bandas, de acordo com a Eq. 1. Para o sensor ETM+ do satélite Landsat 5 a banda do Infra Vermelho Próximo possui intervalo espectral de 800 a $1100 \mathrm{~nm}$ e a banda do Vermelho de 600 a $700 \mathrm{~nm}$. O índice foi proposto visando eliminar diferenças sazonais do ângulo do Sol e minimizar os efeitos da atenuação atmosférica observados para dados multitemporais.

$$
\mathrm{NDVI}=\frac{\mathrm{IVP}-\mathrm{V}}{\mathrm{IVP}+\mathrm{V}}
$$

sendo:

NDVI - valor do índice de vegetação da diferença normalizada
IVP - valor da refletância na faixa do infravermelho próximo

V - valor da refletância na faixa do vermelho

Os valores obtidos do NDVI estão contidos em uma mesma escala de valores entre -1 e +1 . Uma peculiaridade atribuída ao NDVI é sua rápida saturação que o torna insensível ao aumento da biomassa vegetal a partir de determinado estágio de desenvolvimento, ou seja, o índice estabiliza em um patamar apresentando um mesmo valor, embora com o aumento da densidade do dossel.

Os dados foram submetidos à análise estatística descritiva calculando-se média, variância, desvio-padrão, máximo, mínimo e coeficiente de variação. Nesta análise descritiva dos dados utilizou-se o aplicativo Minitab 12.2 ${ }^{\circledR}$.

Para caracterização do padrão da variabilidade espacial foi empregada a análise geoestatística (Vieira, 2000). Semivariogramas foram construídos com base no cálculo da semivariância $\gamma(\mathrm{h})$ por meio da Eq. 2:

$$
\breve{\gamma}(\mathrm{h})=\frac{1}{2 \mathrm{~N}(\mathrm{~h})} \sum_{\mathrm{i}=1}^{\mathrm{N}(\mathrm{h})}\left[\mathrm{Z}\left(\mathrm{x}_{\mathrm{i}}\right)-\mathrm{Z}\left(\mathrm{x}_{\mathrm{i}}+\mathrm{h}\right)\right]^{2}
$$

em que:

$\gamma(\mathrm{h})$ - semivariância estimada a partir dos dados experimentais

$\mathrm{Z}$ - valores medidos nos pontos $\mathrm{x}_{\mathrm{i}}$ e $\mathrm{x}_{\mathrm{i}}+\mathrm{h}$

$\mathrm{N}(\mathrm{h})$ - número de pares de valores medidos separados por uma distância $h$

Os modelos foram ajustados por meio do aplicativo $\mathrm{GS}+\mathrm{v} .7 .0^{\circledR} \mathrm{e}$, em caso de dúvida entre mais de um modelo para o mesmo semivariograma, utilizou-se a técnica de validação cruzada. Observada a dependência espacial entre as amostras, definida pelo semivariograma, é possível estimar valores em que a variável não foi medida fazendo-se uso da krigagem, segundo Vieira et al. (2002).

No sentido de averiguar a correlação entre os atributos em estudo foram construídos semivariogramas cruzados, que podem assumir valores tanto positivos, indicando que o aumento em um dos atributos é acompanhado pelo aumento do outro, como negativos indicando que, quando ocorre o aumento de um dos atributos, ocorre também decréscimo do outro (Bhatti et al., 1991). A confecção dos mapas de krigagem foi feita utilizando-se o programa $\operatorname{Surfer}^{\mathbb{R}}$ v.8.0.

A análise do grau de dependência espacial dos atributos foi realizada segundo Cambardella et al. (1994) em que são considerados de dependência espacial forte os semivariogramas que têm efeito pepita menor ou igual a $25 \%$ do patamar, moderada entre 25 e $75 \%$ e fraca quando for maior que $75 \%$.

\section{Resultados E Discussão}

Os resultados referentes à análise estatística descritiva dos atributos do solo na área em estudo, na camada superficial de 0 a $0,10 \mathrm{~m}$ e do índice de vegetação, podem ser observados nos valores da média, desvio padrão, coeficiente de variação, mínimo e máximo (Tabela 1). 
Tabela 1. Estatística descritiva para argila, fósforo (P), pH e NDVI e correlação dos atributos do solo com NDVI

\begin{tabular}{lcccccc}
\hline Atributos & Média & Desvio padrão & CV (\%) & Mínimo & Maximo & Correlação com NDVI \\
Argila $(\%)$ & 16,16 & 3,24 & 20,01 & 5,95 & 24,85 & $0,27^{*}$ \\
P $\left(\mathrm{mg} \mathrm{dm}^{-3}\right)$ & 4,68 & 1,32 & 28,27 & 3,00 & 11,00 & $0,00^{\text {ns }}$ \\
pH & 4,97 & 0,37 & 7,47 & 4,10 & 6,00 & $0,18^{* *}$ \\
NDVI & 0,40 & 0,05 & 13,09 & 0,21 & 0,49 & \\
\hline
\end{tabular}

ns não significativo; * significativo a 0,$05 ;$ ** significativo a 0,10

A classe analisada de NDVI na imagem Landsat 5/ETM+ da área de cana-de-açúcar apresenta uma amplitude de valores entre 0,21 a 0,49 , com média de 0,40 e desvio-padrão de 0,05 . Esses valores são considerados médios e representam áreas com menor porte vegetativo e mais dessecadas (Weir \& Herring, 2012) corroborando com Lucas \& Schuler (2007) que concluíram que o NDVI da cana-de-açúcar começa a cair a partir do sétimo mês do ciclo da cultura. Ramme (2008) observou, analisando diferentes variedades de cana-de-açúcar, que as diferenças entre solos, estágios de corte e variedades, influenciam na forma de curva do perfil temporal do NDVI sendo mais visualmente perceptível na medida em que essas diferenças são combinadas porém Lopes et al. (2011) concluíram que existe uma relação direta da densidade da vegetação da cana-de-açúcar com o NDVI.

$\mathrm{Na}$ classificação para o coeficiente de variação $(\mathrm{CV})$, os valores obtidos do NDVI apresentaram baixa variabilidade $(13,09 \%)$ segundo a classificação proposta por Cambardella et al. (1994). A medida estatística CV permite, assim, comparar a variabilidade entre amostras de variáveis com unidades diferentes.

Os coeficientes de correlação linear simples (Tabela 1) entre os atributos do solo e o índice de vegetação (NDVI) demonstraram uma correlação positiva e significativa para argila e $\mathrm{pH}$ porém não significativa para a variável fósforo $(\mathrm{P})$.

A.

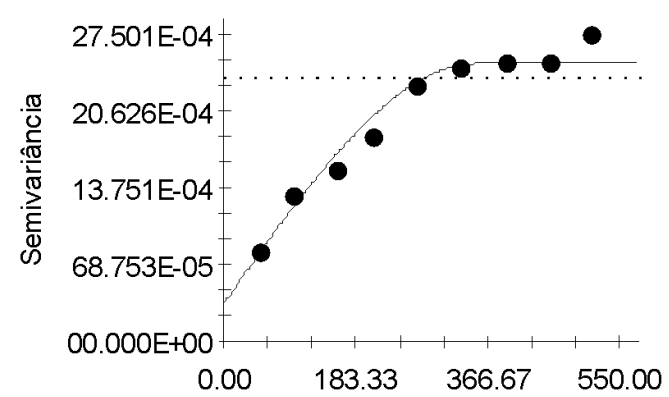

C.

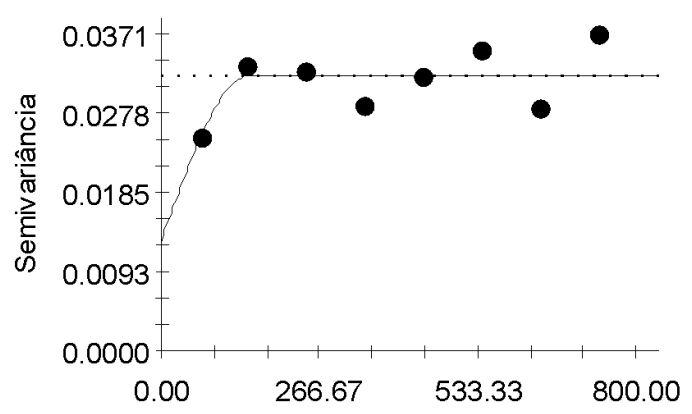

Sendo assim, para correlacionar o NDVI aos atributos do solo justifica-se a análise da dependência espacial para melhor entendimento do padrão de ocorrência desses atributos no espaço. Ressalta-se que uma imagem do mesmo sensor em outra data pode influenciar com resultados diferentes da análise.

Desta forma, para a avaliação da variabilidade e dependência espacial dos atributos estudados foram estimados semivariogramas experimentais isotrópicos dos atributos que apresentaram dependência espacial. O modelo que melhor se ajustou aos semivariogramas experimentais para as variáveis estudadas foi o esférico (Tabela 2 e Figura 2). Este modelo é o mais utilizado para a descrição de variáveis relacionadas às ciências do solo, característico de variáveis que apresentam mudanças abruptas no espaço.

As variáveis estudadas apresentaram diferentes alcances de dependência espacial sendo que a porcentagem de argila

Tabela 2. Modelos e parâmetros estimados dos semivariogramas ajustados aos dados dos atributos do solo e índice de vegetação estudados

\begin{tabular}{lccccc}
\hline Atributo & Modelo & $\mathbf{C}_{\mathbf{0}}$ & $\mathbf{C}_{\mathbf{0}}+\mathbf{C}_{1}$ & $\mathbf{A}(\mathbf{m})$ & $\mathbf{C}_{\mathbf{0}} / \mathbf{C}_{\mathbf{0}}+\mathbf{C}_{\mathbf{1}}$ \\
Argila & esférico & $3,27 \mathrm{E}+00$ & $1,08 \mathrm{E}+01$ & 403,21 & 0,30 \\
$\mathrm{P}$ & esférico & $1,28 \mathrm{E}-02$ & $2,70 \mathrm{E}-02$ & 148,38 & 0,47 \\
$\mathrm{pH}$ & esférico & $6,93 \mathrm{E}-02$ & $1,47 \mathrm{E}-01$ & 243,54 & 0,47 \\
$\mathrm{NDVI}$ & esférico & $3,36 \mathrm{E}-04$ & $2,49 \mathrm{E}-03$ & 352,13 & 0,14 \\
\hline
\end{tabular}

$\mathrm{P}$ - Fósforo; $\mathrm{C}_{0}$ - Efeito pepita; $\mathrm{C}_{0}+\mathrm{C}_{1}$ - Patamar; $\mathrm{A}$ - Alcance

B.

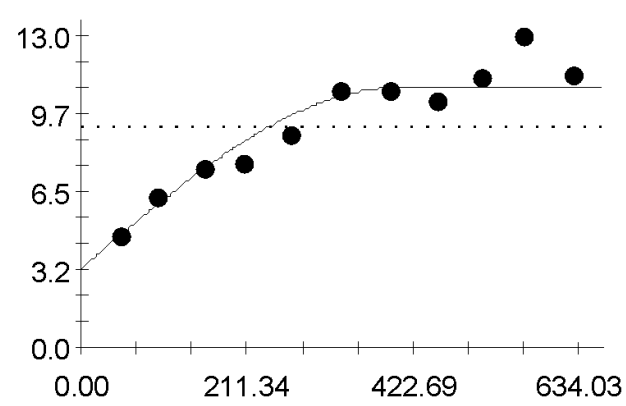

D.

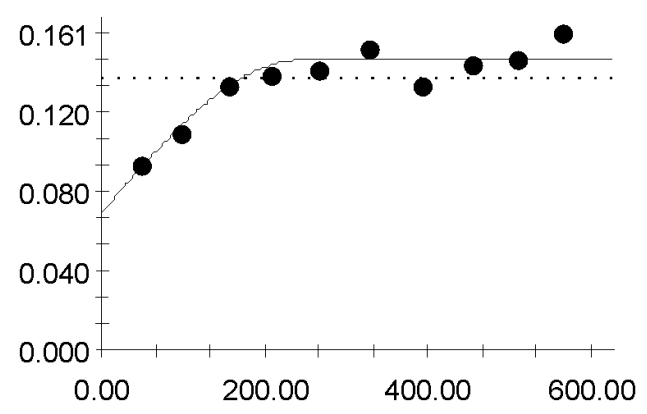

Distância de Separação $(\mathrm{m})$

Figura 2. Semivariogramas das variáveis estudadas: NDVI (A), Argila (B), Fósforo (C) e pH (D) 
apresentou maior alcance $(403,21 \mathrm{~m})$ e o fósforo menor alcance (148,38 m) (Tabela 2, Figura 2). Ferraz et al. (2012) também encontraram, em um Latossolo vermelho-amarelo, valores de alcance próximos a $140 \mathrm{~m}$ para fósforo.

Analisando a relação $\mathrm{C}_{0} /\left(\mathrm{C}_{0}+\mathrm{C}_{1}\right)$ em percentagem dos atributos argila, fósforo e $\mathrm{pH}$, isoladamente, esta indica grau de dependência espacial moderado $\left[\mathrm{C}_{0} /\left(\mathrm{C}_{0}+\mathrm{C}_{1}\right)\right.$ entre 25 e $75 \%$ ] segundo a classificação proposta por Cambardella et al. (1994) (Tabela 3). Souza et al. (2004) também observaram ocorrência de dependência espacial moderada para argila em um Latossolo sob cultivo de cana-de-açúcar.

Para o índice de vegetação (NDVI), a relação $\mathrm{C}_{0} /\left(\mathrm{C}_{0}+\mathrm{C}_{1}\right)$ em percentagem foi de $14 \%$ indicando um grau de dependência espacial forte, segundo a classificação citada.

Nos mapas de distribuição espacial obtidos observa-se que o padrão de distribuição espacial do NDVI (Figura 3A) é semelhante à distribuição espacial das variáveis argila (Figura 3B) e fósforo (Figura 3C) o que também é comprovado quando se analisam esses atributos do solo, correlacionados
A.

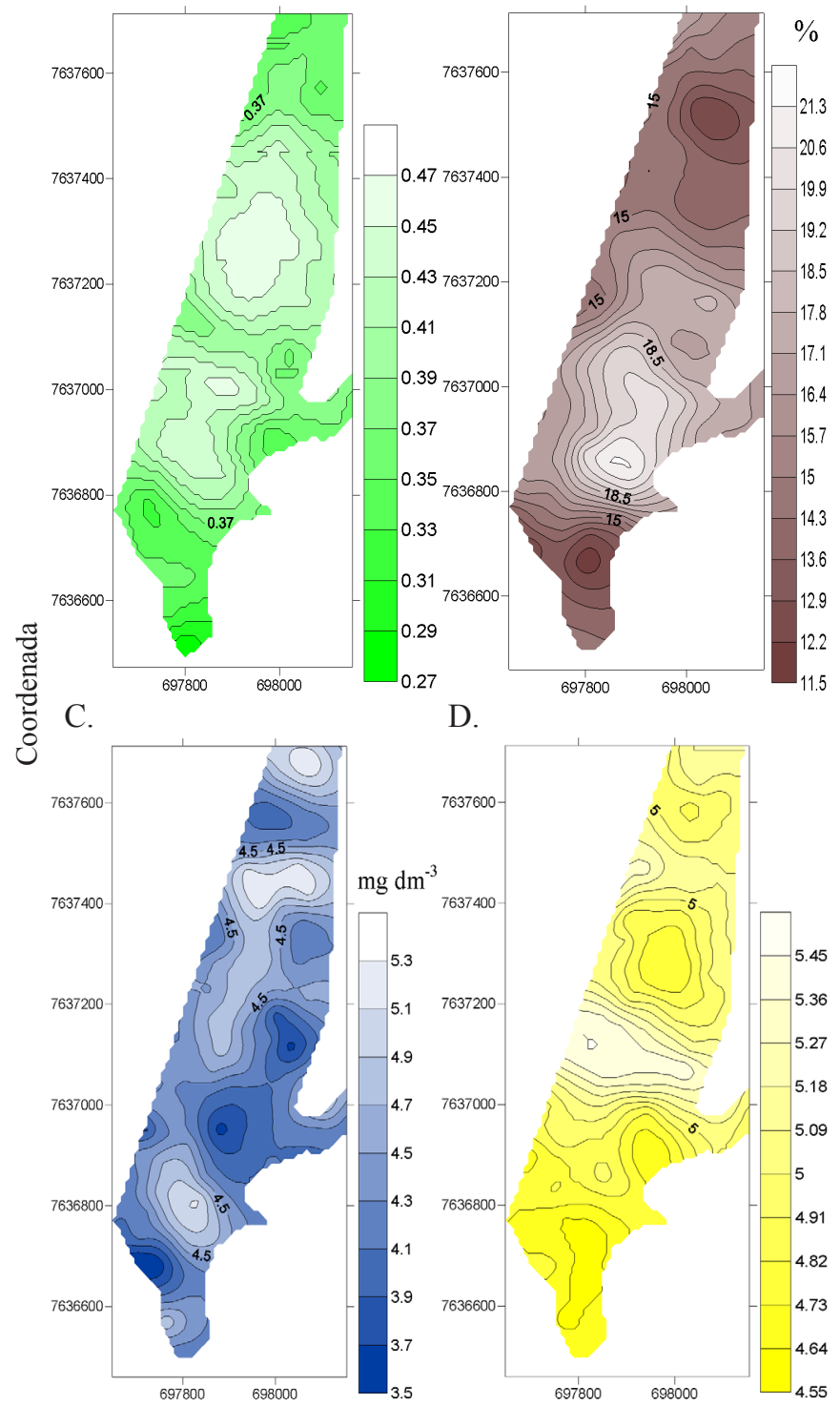

Figura 3. Mapas do padrão espacial das variáveis estudadas: NDVI (A), Argila (B), Fósforo (C) e pH (D) com o NDVI na imagem Landsat a relação $\mathrm{C}_{0} /\left(\mathrm{C}_{0}+\mathrm{C}_{1}\right)$ dos semivariogramas cruzados mostra valores $<25 \%$ (Tabela 3 ) que, conforme Cambardella et al. (1994) é classificado como sendo grau de dependência espacial forte, indicando que mesmo não havendo correlação linear significativa entre o NDVI e o fósforo (Tabela 1), há correlação espacial entre esses atributos (Tabela 3) com grau de dependência forte justificando o estudo da correlação espacial, independentemente da correlação linear.

Tabela 3. Modelos e parâmetros estimados dos semivariogramas cruzados ajustados aos dados dos atributos do solo em correlação com o índice de vegetação estudado

\begin{tabular}{lccccc}
\hline Atributo & Modelo & $\mathbf{C}_{\mathbf{0}}$ & $\mathbf{C}_{\mathbf{0}}+\mathbf{C}_{1}$ & $\mathbf{A}(\mathbf{m})$ & $\mathbf{C}_{\mathbf{0}} / \mathbf{C}_{\mathbf{0}}+\mathbf{C}_{\mathbf{1}}$ \\
Argila & esférico & $8,91 \mathrm{E}-03$ & $4,46 \mathrm{E}-02$ & 301,17 & 0,20 \\
$\mathrm{P}$ & esférico & $1,00 \mathrm{E}-05$ & $1,25 \mathrm{E}-03$ & 145,53 & 0,01 \\
$\mathrm{pH}$ & $\mathrm{EPP}$ & & & & \\
\hline
\end{tabular}

$P$ - Fósforo, $C_{0}$ - Efeito pepita; $C_{1}$ - Patamar; A - Alcance; EPP - efeito pepita puro

Carvalho et al. (2004) também encontraram variabilidade espacial nos atributos do solo e concluíram que a distribuição espacial não foi ao acaso e sim resultante de processos naturais e de práticas culturais. A avaliação da variabilidade espacial dos atributos estudados mostra que, mesmo na classe dos Latossolos sob cultivo de cana-de-açúcar por longo tempo, pode-se registrar variabilidade espacial.

$\mathrm{Na}$ Figura 4 pode-se observar a correlação espacial positiva do índice de vegetação (NDVI), com a \% de argila e teor de

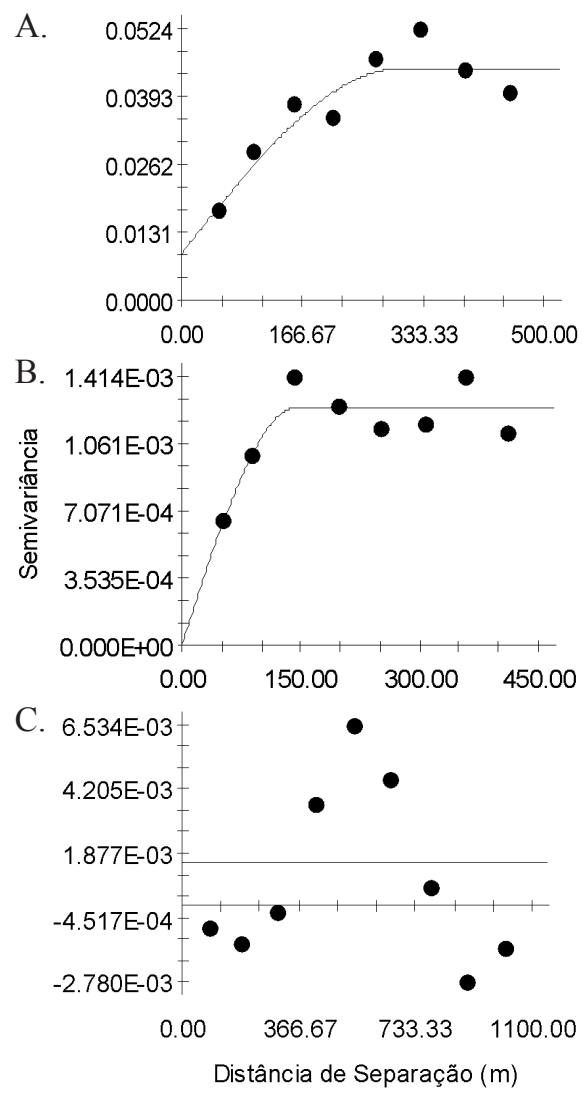

Figura 4. Semivariogramas cruzados dos atributos estudados: \% de Argila em função do NDVI (A), teor de fósforo em função do NDVI (B) e pH em função do NDVI (C) 
fósforo no solo porém não houve correlação espacial entre o NDVI e o pH do solo; o efeito pepita puro mostra que não há dependência espacial correlacionada para essas duas variáveis.

Lourenço (2005) concluiu, relacionando os atributos do solo e da planta com a resposta espectral da cana-de-açúcar, que a porcentagem explicada da resposta espectral NDVI para os dois anos estudados foi satisfatória haja vista a ausência dos demais atributos modelos, podendo a explicação ter sido maior caso tivessem sido incluídas, na análise, outras variáveis relacionadas ao clima e ao solo.

Imagens de sensores remotos com diferentes resoluções espaciais, radiométricas e temporais, podem propiciar constatação de correlação semelhante conforme apresentado neste trabalho. Entretanto, trabalhos relacionados à correlação desses índices de vegetação com fatores edáficos, ainda são escassos (Weir \& Herring, 2012). Kariyeva \& Leeuwen (2011) identificaram variáveis ambientais da relação solo-paisagem, como relevo, escoamento superficial das águas das chuvas, teor de nitrogênio do solo e densidade do solo. Os valores avaliados apresentaram correlação com o NDVI demonstrado a eficácia na utilização de imagens de satélites para análise ambiental; apesar disto, ocorre uma restrição na análise da correlação conforme metodologia apresentada no trabalho, decorrente de ruídos (exemplo nuvens). A imagem a ser utilizada deverá ser tratada para que não ocorra interferência nos resultados correlacionados

\section{CONClusões}

1. Os atributos que apresentaram correlação espacial com o NDVI na área de estudo, foram argila e fósforo.

2. $\mathrm{O}$ pH do solo não apresentou correlação espacial com o índice de vegetação estudado.

3. As imagens do Landsat 5/ETM+ da data estudada em relação ao NDVI da cana-de-açúcar apresentaram boa aplicação para observar a variabilidade espacial dos atributos estudados.

\section{Agradecimentos}

À FAPESP, pela bolsa de estudos, e ao Grupo Virgolino de Oliveira-Usina Catanduva, pelo apoio e concessão da área de estudo.

\section{Literatura Citada}

Bhatti, A. U. Mulla, D. J.; Frazier, B. E.Estimation of soil properties and wheat yields on complex eroded hills using geostatistics and thematic mapper images. Remote Sensing of Environment, v.37, p.181-191, 1991.

Cambardella, C. A.; Moorman, T. B.; Novak, J. M.; Parkin, T. B.; Karlen, D. L; Turco R.F.; Konopka, A. E. Field scale variability of soil properties in central Iowa soils. Soil Science Society of America Journal, v.58, p.1501-1511, 1994.

Carvalho, J. R. P.; Dechen, S. C. F.; Dufranc, G. Variabilidade espacial da agregação do solo avaliada pela geometria fractal e geoestatística. Revista Brasileira de Ciência do Solo, v.2, p.1-9, 2004.
Cohen, W. B.; Maiersperger, T. K.; Gower S. T.; Turner, D. P. An improved strategy for regression of biophysical variables and Landsat ETM+ data. Remote Sensing of Environment, v.84, p.561-571, 2003.

Dorigo, W. A.; Milla, R. Z.; de Wit, A.J.W.; Brazile, J.; Singh, R.; Schaepman, M.E. A review on reflective remote sensing and data assimilation techniques for enhanced agroecosystem modeling. International Journal of Applied Earth Observation and Geoinformation, v.9, p.165-193, 2007.

Ferraz, G. A. E. S.; Silva, F. M. da; Carvalho, L. C. C.; Alves, M. de C.; Franco, B. C.Variabilidade espacial e temporal do fósforo, potássio e da produtividade de uma lavoura cafeeira. Engenharia Agrícola, v.32, p.140-150, 2012 .

IBGE - Instituto Brasileiro de Geografia e Estatística. 2012. $<$ http://www.ibge.gov.br/home/estatistica/indicadores/ agropecuaria/lspa/default.shtm. $10 \mathrm{Fev} 2012$.

Johnsen, G. A.; Mortensen, D. A.; Gotway, C. A. Spatial and temporal analysis of weed seedling populations using geostatistics. Weed Science, v.44 p.704-710, 1996.

Johnson, L. F.; Trout, T. J. Satellite NDVI assisted monitoring of vegetable crop evapotranspiration in California's San Joaquin valley. Remote Sensing,v.4, p.439-455,2012.

Kariyeva, J.; Leeuwen, W. J. D. van. Environmental drivers of NDVI- Based vegetation phenology in Central Asia. Remote Sensing, v.3, p.203-246, 2011.

Lopes, H. L.; Accioly, L. J. de O.; da Silva, F. H. B. B.; Sobral, M. do C. M.; Araújo Filho, J. C. de; Candeias, A. L. B. Espacialização da umidade do solo por meio da temperatura da superfície e índice de vegetação. Revista Brasileira de Engenharia Agrícola e Ambiental, v.15, p.973-980, 2011.

Lourenço, L. S. Aplicação da estatística multivariada no estudo da relação entre atributos do solo e da planta e a resposta espectral da cana-de-açúcar. Campinas: FEAGRI/ UNICAMP, 2005.134p. Dissertação Mestrado

Lucas, A. de A.; Schuler, C. A. B. Análise do NDVI/NOAA em cana-de-açúcar e Mata Atlântica no litoral norte de Pernambuco. Revista Brasileira de Engenharia Agrícola e Ambiental, v.11, p.607-614. 2007.

McBratney, A. B.; Pringle, M. J. Estimating average and proportional variograms of soil properties and their potential use in precision agriculture. Precision Agriculture, v.1, p.219-236, 1999.

Miura, T.; Huete, A. R.; Yoshioka, H.; Holben, B. N. An error and sensitivity analysis of atmospheric resistant vegetation indices derived from dark target-based atmospheric correction. Remote Sensing of Environment, v.78, p.284298, 2001.

Ponzoni, F. J. Comportamento espectral da vegetação. In. Sensoriamento Remoto - Reflectância dos alvos naturais. Brasília: UnB, EMBRAPA, 2001. p.157-199.

Ramme, F. L. P. Perfis temporais NDVI e sua relação com diferentes tipos de ciclos vegetativos da cultura da canade-açúcar. Campinas: FEAGRI/UNICAMP, 2008. 103p. Tese Doutorado 
Souza, Z. M.; Marques Júnior, J.; Pereira, G. T.; Barbieri, D. M. Variabilidade espacial da textura de um Latossolo Vermelho eutroférrico sob cultivo de cana-de-açúcar. Engenharia Agrícola, v.24, p.309-319, 2004.

Trangmar, B. B.; Yost, R.S.; Wade, M. K.; Uehara, G. Applications of geostatistics to spatial studies of soil properties. Advances in Agronomy, v.38, p.45-94, 1985.

Verhulst, N.; Govaerts, B.; Sayre, K. D.; Deckers, J.; François, I. M.; Dendooven, L. Using NDVI and soil quality analysis to assess influence of agronomic management on within-plot spatial variability and factors limiting production. Plant and Soil, v.317, p.45-59, 2009.
Vieira, S. R. Geoestatística em estudos de variabilidade espacial do solo. In: Novais, R. F.; Alvarez V., V. H.; Schaefer, C. E. (ed.) Tópicos em ciências do solo. Viçosa: Sociedade Brasileira de Ciência do Solo, 2000. p.1-54.

Vieira, S. R.; Millete, J.; Topp, G. C.; Reynolds, W. D. Handbook for geostatistical analysis of variability in soil and climate data. In: Alvarez V., V. H.; Schaefer, C. E. G. R.; Barros, N. F.; Mello, J. W. V.; Costa, L.M. (ed.).Tópicos em ciência do solo, Viçosa: SBCS, v.2. 2002. p.1-45.

Weir J.; Hering, D. Measuring vegetation (NDVI \& EVI). NASA Earth Observatory.2012. <http://earthobservatory.nasa.gov/ Features/MeasuringVegetation/measuring_vegetation_3. p>. 10 Mai. 2012. 